\title{
A Threshold based Algorithm to Detect Peripapillary Atrophy for Glaucoma Diagnosis
}

\author{
Jahin Majumdar \\ Computer Science and Engineering Department \\ Amity School of Engineering and Technology \\ Amity University, Noida, India
}

\begin{abstract}
The presence of a Peripapillary Atrophy (PPA) is one of the conditions for Glaucoma to develop. This paper is divided into three parts. The first part of this paper describes the terminology related to the diagnosis of glaucoma. The second part of this paper describes various existing algorithms to detect and segment human PPA from a digital fundus retinal image. The paper compares the performances and contrasts the various shortcomings of these described algorithms. The third part of this paper proposes a threshold-based algorithm to detect the PPA of a human eye to aid the diagnosis of Glaucoma. The proposed algorithm calculates the Red by Green ratio for each pixel in the Region of Interest (ROI) and segments the Optic Disc (OD) from the PPA, having different pixel ratios. The algorithm can be further improved by applying sub-algorithms of false region elimination. The proposed algorithm should, theoretically, overcome most of the problems faced by the described algorithms.
\end{abstract}

\section{General Terms}

Glaucoma, Peripapillary Atrophy, Red-by-Green ratio, Medical Image Segmentation, Fundus image.

\section{Keywords}

Glaucoma diagnosis, Peripapillary Atrophy, Optic Disc, Optic Cup.

\section{INTRODUCTION}

Glaucoma is a cause of blindness that affects a majority of people in the world. It is estimated that, in developed countries, fewer than $50 \%$ of those with glaucoma are aware of their disease [1]. It is due to this unawareness that Glaucoma is so prevalent. Existing diagnosis methods like Tonometry [3], Ophthalmoscopy [4] and Perimetry [5] are cumbersome and time-consuming for the patient and has significant error rates. So, a technique is needed to detect Glaucoma automatically by analyzing the digital fundus retinal image of a human eye. Glaucoma occurs due to a variety of reasons that will be explained later in subsequent paragraphs.

One of the most important methods to detect a glaucomatous eye is to detect the presence of a PPA in the eye. PPA occurs around the OD, which in turn surrounds the OC. The traditional methods include segmenting the OD and the OC from the fundus image and calculating the Cup-to-Disc Ratio (CDR) [7] [8]. If the ratio is beyond 0.3, it is concluded that it is a glaucomatous eye. However, the above situation fails when a PPA is present in the eye. In such a scenario, the segmented OD includes the PPA due to similar intensity. This inclusion increases the (OD + PPA) area, thereby decreasing the CDR. This can prove to be harmful to patients due to false diagnosis. Hence, a method is required to detect the PPA successfully and prevent it from appearing as a part of the OD. There are some existing methods to segment the PPA, which have been described in Section 2. The main contribution of this paper is to minimize various shortcomings of previous techniques. The proposed algorithm uses a threshold in the RGB (Red Green Blue) matrix whose threshold value can be changed for different database of images, keeping the algorithm intact. It repeatedly calculates the $\mathrm{R} / \mathrm{G}$ ratio for each pixel and selects those pixels whose value goes beyond a threshold.

\section{RELATED WORK}

The work related to detection and segmentation of PPA is limited. The work that has been done so far and will be mentioned in this paper includes techniques of feature extraction, texture analysis, edge filtering and detection and some threshold based techniques. C. Muramatsu et al. [9] describe a method involving texture analysis but produce moderate classification accuracy. J. Cheng et al. [10] used edge detection followed by edge filtering but uses unreliable OC segmentation techniques. J. Cheng et al. [11] provided another algorithm for PPA detection using biologically inspired feature. J. Cheng et al. [12] provided yet another improved algorithm for PPA detection by sparse biologically inspired feature manifold. A detailed study of these works will be presented in Section 4.

\section{BACKGROUND}

\subsection{Causes and Diagnosis of Glaucoma}

Glaucoma occurs when the drainage channels in the eye are partially or wholly blocked, preventing the proper drainage of eye fluids. This blockage causes the fluids to accumulate in the back of the eye causing a pressure to the Optic Nerve Head. The exact cause of this blockage is unknown. This condition, if untreated can lead to permanent blindness. Glaucoma can be diagnosed by analyzing three major conditions within the eye.

\subsubsection{CDR}

The pressure causes the $\mathrm{OC}$ to increase its diameter vertically while the OD remains constant. Thus, the ratio between an $\mathrm{OC}$ and $\mathrm{OD}$ will be different in a normal eye and a glaucomatous eye. There are two ways to find out this ratio. Firstly, by calculating the area of OD and OC and finding the ratio (Area $\mathrm{OC}_{\mathrm{OC}} / \mathrm{Area}_{\mathrm{OD}}$ ). Secondly, by finding the vertical diameter of both the $\mathrm{OD}$ and $\mathrm{OC}$ and finding the ratio $\left(\mathrm{VD}_{\mathrm{OC}} /\right.$ $\left.\mathrm{VD}_{\mathrm{OD}}\right)$. If the ratio is above 0.3 , the eye can be diagnosed as Glaucomatous.

\subsubsection{NRR}

Neuroretinal Rim (NRR) area is the second method for diagnosing Glaucoma [13]. NRR is the region outside the OC surrounding the OD. As the size of the OC increases, the area 
of the NRR decreases. Thus, the reduction in the area of the NRR can be compared to a normal eye and a Glaucomatous eye. The affected eye will have a smaller NRR.

\subsubsection{ISNT}

ISNT - This is the third condition required for the diagnosis of Glaucoma. Interior Superior Nasal Temporal (ISNT) is a rule that states that the optic nerve will be thickest Inferiorly, followed by Superiorly, and then Nasally and finally thinnest Temporally. This rule applies to blood vessels and OC also. A glaucomatous eye will not follow this rule, but a normal eye will. Thus, Glaucoma can be diagnosed easily by analyzing whether this ISNT rule is broken or followed. Normally, there are five conditions for a non-glaucomatous eye:

1) The OC / OD Ratio should be less than or equal to 0.3.

2) The Neuroretinal Rim (NRR) should be large enough.

3) Blood vessels, OC and optic nerve, should follow ISNT.

4) Peripapillary Atrophy should be absent, be it $\alpha$ or $\beta$.

5) There should be no hemorrhage around 25\% area of OD.

\subsection{Optic Disc}

The Optic Disc is one of the most distinctive features visible in a digital retinal fundus image, according to [14] and [15]. The optic disc marks the region where the blood vessels and the optic nerve originate and reach the retina. It is the optic disc that transports information from the eye to the brain. In the fundus image of a human retina, the optic disc appears as a bright, yellow elliptical or circular region. Its position varies from eye to eye. The blood vessels can be seen as emerging from the optic disc. The size of the optic disc remains constant for a normal eye and a glaucomatous eye. However, most optic disc segmentation techniques include the PPA along with optic disc due to similar intensity and brightness. This inclusion makes the optic disc falsely appear as large. It is a significant factor in determining the cup-to-disc (CDR) ratio.

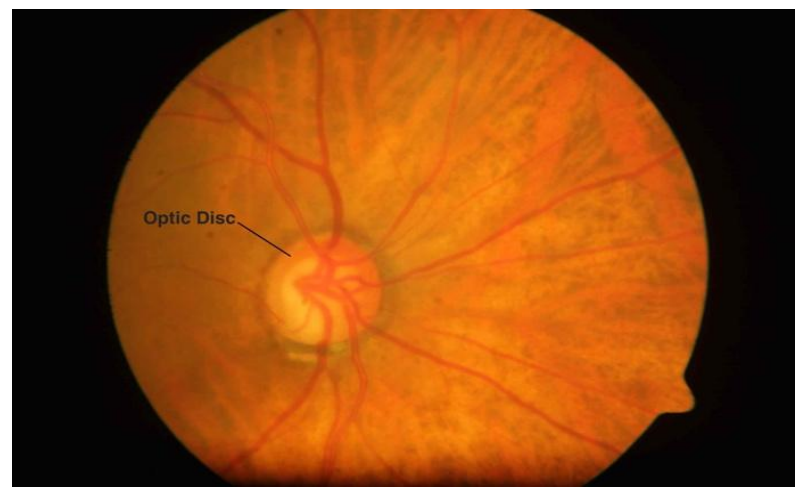

Figure 1: A digital retinal fundus image showing the optic disc

\subsection{Optic Cup}

The Optic Cup (OC) is another major feature in a digital fundus retinal image. It lies inside the optic disc. It usually appears brighter than the optic disc in the fundus image. The shape and area of the optic cup vary from a normal eye to a glaucomatous eye. It increases in its area when a patient is affected by glaucoma, due to the accumulation of fluid in the region. When it increases in size, its shape becomes irregular. The optic cup can be studied and is useful in finding out the cup-to-disc ratio to diagnose glaucoma.

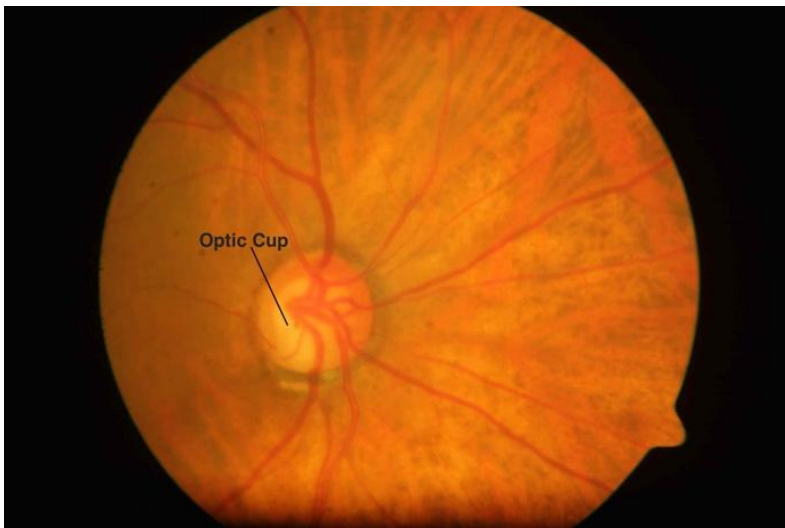

Figure 2: A digital retinal fundus image showing the optic cup

\subsection{Peripapillary Atrophy}

Peripapillary atrophy (PPA) refers to the region surrounding the optic disc. According to S. Talu et al. [15], PPA is of pigmented crescent-shaped. The presence of PPA denotes that the patient has Glaucoma. In severe cases, the PPA surrounds the whole optic disc. PPA can be divided into two categories, namely:

1) $\alpha$ - PPA: This type of PPA occurs during the initial stages of Glaucoma. It causes the pigmentary and structural irregularity of retinal pigment epithelial (RPE) cells. This type of PPA appears to be darker in comparison to the optic disc. 2) $\beta$ - PPA: This type of PPA occurs during the later and final stages of Glaucoma. It causes the complete loss of retinal pigment epithelial (RPE) cells along with the variable loss of photoreceptor cells according to [14]. This type of PPA appears to be white in comparison to the optic disc.

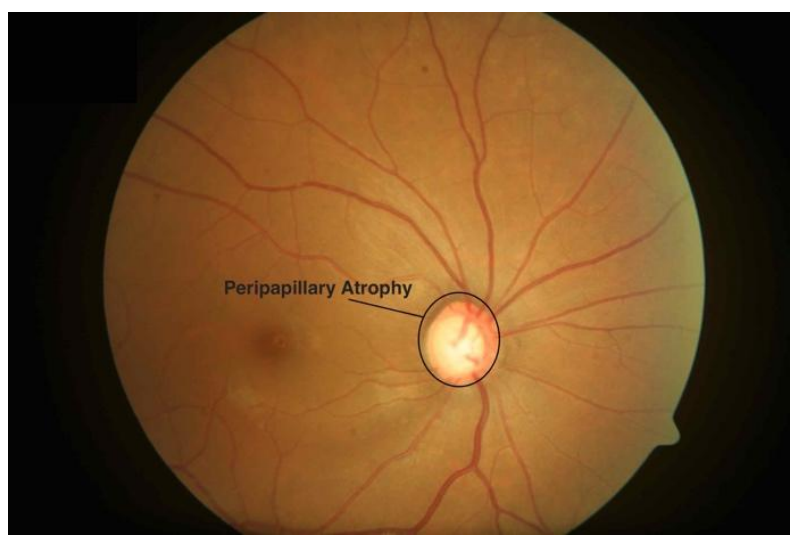

Figure 3: A digital retinal fundus image showing PPA

\section{EXISTING TECHNIQUES 4.1 Edge Detection and Filtering Technique}

J. Cheng et al. [10] proposed this technique wherein they initially used localization algorithms and the fringe removal based method to get the region of interest (ROI). They use a 2D mean filter to smoothen the image. The smoothed image is then considered, and its derivative for each row profile is calculated. The local maxima and minima will be the boundaries of the optic disc. After detecting the edges, edge filtering is applied.

In the process of edge filtering, the ROI is compared with a threshold and processed with a morphological closing 
operation. Cheng et al. used a morphological dilation function. The threshold value used was initialized to the mean intensity of the ROI and set to an empirically determined brightness intensity. This technique, however, excludes edges only from the $\alpha$ PPA. The blood vessels are also removed that correspond to the local minima.

The edge filtering is followed by an approximation of the optic disc by a constrained elliptical Hough transformation. This approximation technique enables the PPA to be detected more accurately. The paper states that if $b_{1} / a_{1}>\gamma$, the probability that the detected ellipse contains the PPA is high. If $\mathrm{b} / \mathrm{a} \leq \gamma$, it can be concluded that the PPA has been excluded. To detect $\beta$ PPA, the authors empirically determine the disc boundary. The feature points are extracted, and if they exceed a threshold level in a quadrant, $\beta$ PPA is said to be present.

The technique proposed by J. Cheng et al. [10] is significantly accurate and can be improved by further using ellipse fitting correction techniques.
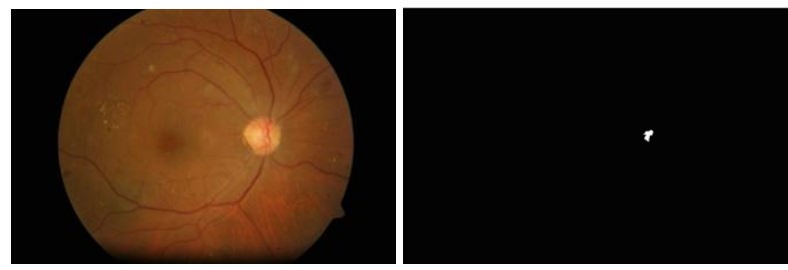

Figure 4: A digital retinal fundus image showing the segmented Optic Cup

\subsection{Texture Analysis Technique}

C. Muramatsu et al. [9] proposed an algorithm to detect the PPA in the fundus image by extracting the required textures and features. The initial procedure involves segmenting the optic disc using a p-tile thresholding method. Some features such as Contrast, Correlation, Variance, Inverse difference moment, Entropy and Dissimilarity were extracted from the grey level co-occurrence (GLCM) matrix.

Followed by the texture analysis, the PPA and the non-PPA pixels were classified using a linear discriminant analysis (LDA). The next step involved binarization of the ROIs with an empirically determined threshold. The authors estimated that since, the PPA will be present around the OD boundary, the fraction of pixels greater than $3 \%$ that were in contact with the optic disc boundary will be considered as PPA pixels. The optic disc boundary was detected using Canny Edge detection.

The technique produces a specificity of $73 \%$. The technique described is particularly helpful for detecting severe PPAs but does not give accurate results for mild PPA. In that case, PPA pixels are considered as normal pixels and a false result is obtained. This failure is mainly due to the suboptimal performance of LDA in the training data set. The sensitivity should be improved to produce an accurate result.

\subsection{Biologically Inspired Feature Technique}

J. Cheng et al. [11] proposed another method to detect PPA by mimicking the technique of cortex for visual perception. The focal region of the eye is segmented from the retinal image, and the biologically inspired feature is extracted.

The biologically inspired feature (BIF) consists of intensity units that are obtained from a convolution function involving dyadic Gaussian pyramids and the color image's intensity channel. The BIF also includes color units that are generated by nine spatial scales per color channel. Two color pairs are considered, and the surround maps are interpolated to be the same structure as the center maps. Lastly, the BIF includes C1 units pooled over S1 units. The paper uses the Gabor mother function that are pooled over adjacent scales.

The process of focal region classification and segmentation is performed to reduce the computational time of the algorithm. This is based on the assumption that human examiners tend to focus on the focal region. The paper proposes a threshold function to segment the focal region and detect the optic disc. The adaptive threshold function locates the bright spots that will be the optic disc. Similar to the previous paper, this technique again uses a constrained elliptical Hough transformation to smoothen the boundary of the optic disc.

The paper states that this technique of PPA segmentation achieves a 90\% accuracy. This technique is independent of the size of the PPA and the accuracy increases with the width of the PPA.
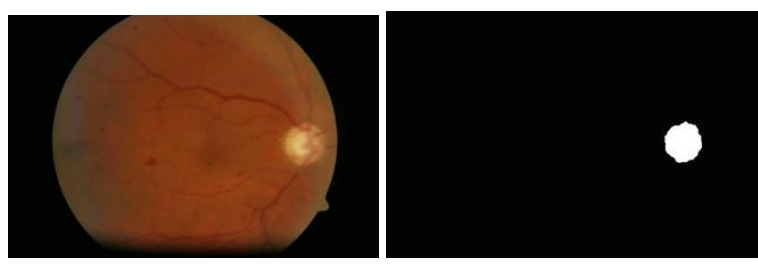

Figure 5: A digital retinal fundus image showing the segmented Optic Disc (includes PPA)

\subsection{Sparse Biologically Inspired Feature Manifold Technique}

This is yet another method by J. Cheng et al. [12] which uses biologically inspired feature with sparse transfer learning. This algorithm is similar to the previous paper [11] except the fact that it uses a sparse transfer learning to reduce redundancy.

A linear mapping tool is used followed by a formulation of sparse transfer learning. Here, the authors attempt to maximize the distance between like samples and minimize the distance between dissimilar samples. The Sample distribution knowledge to obtain balanced training samples with the same class size. Finally, Elastic Net Penalty is used according to regularization theory to control the model complexity.

This algorithm achieves significantly greater accuracy compared to other algorithms. The BIF technique achieves an average accuracy of $87 \%$. Similarly, the adaptive threshold, the negative sparse transfer learning and the focal region segmentation achieves desirable precision. It concludes that $\mathrm{nSTL}$ is superior to pSTL in PPA elimination and detection.

This algorithm uses machine learning tools and techniques along with image processing algorithms. It achieves the highest accuracy compared to the other three algorithms described. Its limitation includes the computation of the extent and progression of PPA. 
Table 1: An overview of the existing PPA detection algorithms

\begin{tabular}{|l|l|l|}
\hline Title of Paper & Author & Technique Used \\
\hline $\begin{array}{l}\text { Automatic Optic Disc Segmentation with } \\
\text { Peripapillary Atrophy Elimination }\end{array}$ & $\begin{array}{l}\text { J. Cheng, J. Liu, D. W. K. } \\
\text { Wong, F. Yin, C. Cheung, M. } \\
\text { Baskaran, T. Aung, T. Y. } \\
\text { Wong }\end{array}$ & $\begin{array}{l}\text { The technique used involves edge detection and } \\
\text { edge filtering with a constrained elliptical Hough } \\
\text { transform }\end{array}$ \\
\hline $\begin{array}{l}\text { Computerized Detection of Peripapillary } \\
\text { Chorioretinal Atrophy by Texture Analysis }\end{array}$ & $\begin{array}{l}\text { C. Muramatsu, Y. Hatanaka, A. } \\
\text { Sawada, T. Yamamoto, H. } \\
\text { Fujita }\end{array}$ & $\begin{array}{l}\text { This technique uses feature extraction followed } \\
\text { by pixel classification technique by linear } \\
\text { discriminant analysis. }\end{array}$ \\
\hline $\begin{array}{l}\text { Peripapillary atrophy detection } \\
\text { biologically inspired feature by }\end{array}$ & $\begin{array}{l}\text { J. Cheng, J. Liu, D.W.K. } \\
\text { Wong, N. Tan, C. Cheung, M. } \\
\text { Baskaran, T. Y. Wong, Seang } \\
\text { Mei Saw }\end{array}$ & $\begin{array}{l}\text { This method uses Biologically Inspired Feature } \\
\text { (BIF) and a threshold based optic disc } \\
\text { segmentation to detect and segment the PPA }\end{array}$ \\
\hline $\begin{array}{l}\text { Peripapillary Atrophy Detection by Sparse } \\
\text { Biologically Inspired Feature Manifold }\end{array}$ & $\begin{array}{l}\text { J. Cheng, D. Tao, J. Liu, D. W. } \\
\text { K. Wong, N. Tan, T. Y. Wong, } \\
\text { S. M. Saw }\end{array}$ & $\begin{array}{l}\text { This technique is an improved of the BIF } \\
\text { technique and it involves negative sparse transfer } \\
\text { learning for accurate results. }\end{array}$ \\
\hline
\end{tabular}

\section{PROPOSED ALGORITHM}

This section aims to provide a different technique that can be used to detect PPA from a fundus image. It is based on a threshold-based algorithm. However, the threshold is applied to a matrix containing the $\mathrm{R}, \mathrm{G}, \mathrm{B}, \mathrm{Y}$ values of a region of interest (ROI). The algorithm further depends on a threshold based optic disc and optic cup segmentation algorithm [16] and training data set to calculate the Red-by-Green (R/G) ratio range. The algorithm error rate is minimized by false region elimination. This technique is based on the fact that $\mathrm{R} / \mathrm{G}$ ratio is a distinctive feature of human skin and the same can be applied to a human eye. The proposed algorithm should theoretically overcome the difficulties of the previously described algorithms.

\section{METHODOLOGY}

\subsection{Optic Disc Segmentation}

The Optic Disc is segmented from the digital fundus retinal image by considering the red channel. The red channel is preprocessed initially. The pre-processing involves taking a GLCM matrix and initially extracting three features. Various images are then analyzed their features are studied. For those images, whose features lie within a certain range, the mean and the standard deviation of the entire image is subtracted twice from the red channel. For other images, the mean and the standard deviation is subtracted thrice. This gives the preprocessed red channel that is ready for thresholding. The image obtained contains only the optic nerve head. Next, a thresholding function is applied. A Gaussian window is taken into consideration and convoluted with the histogram of the red channel, resulting in a smoothed histogram. This gives the segmented optic disc that is then subjected to morphological operations to reduce the error rate. In PPA images, this optic disc includes the PPA pixels.

\subsection{Optic Cup Segmentation}

The optic cup segmentation is similar to the optic disc segmentation except that it is calculated on a green channel. The mean and the standard deviation of the entire image is subtracted thrice from the green channel to preprocess it. This pre-processed image excludes the blood vessels in the optic cup and is ready for thresholding. The histogram of the preprocessed green channel is convoluted with a Gaussian window to obtain a smoother histogram. After applying the threshold function, the image is again subjected to morphological transformation to improve accuracy. The final image contains the segmented optic cup.

\subsection{Region of Interest (ROI)}

The region of interest is the area that will be considered for PPA detection. As seen from Figure 5, the OD includes the $\mathrm{OC}$ and the PPA. The ROI will contain the remaining area between the (OD+PPA) - OC region. In other words, it will contain the Neuroretinal rim (NRR). The area is subjected to a simple subtraction operation to get the remaining area to obtain the ROI.

\subsection{Constrained Elliptical Hough Transformation (CEHT)}

The area containing the optic disc and PPA is approximated to an ellipse to find out the vertical and horizontal boundary using the CEHT function. The OD+PPA region is approximated to an ellipse according the equation (1):

$$
\frac{(x-h)^{2}}{a^{2}}+\frac{(y-k)^{2}}{b^{2}}=1
$$

The approximation gives the vertical diameter ' $a$ ' and the horizontal diameter ' $b$ ' which will be the upper boundary of the ROI.

\subsection{Red-By-Green Ratio Calculation}

After the CEHT function is applied, the original image is once again considered. The ROI with the horizontal and vertical diameter is taken as the limit, and an RGB matrix is calculated for that region. This is followed by calculating the ratio of the red channel value and the green channel value.

\subsubsection{Training Dataset}

In the training dataset, PPA affected images were considered where all the images contain $\alpha$ or $\beta$ PPA. The R/G ratio is found out repeatedly for each image and the maximum, and the minimum value is noted. This value will provide the range of $\mathrm{R} / \mathrm{G}$ ratios that act as the upper and lower limit threshold value.

\subsubsection{Test Dataset}

The threshold range found out in the training dataset will be used to detect the PPA pixels in the test dataset. The same technique is applied and the $\mathrm{R} / \mathrm{G}$ ratio of all the pixels stored in the matrix is calculated. If any pixel lies below the minimum threshold or above the maximum threshold, it is set 
to white. The other pixels are set to black. Those pixels are classified as PPA pixels. This is based on the assumption that the $\mathrm{R} / \mathrm{G}$ ratio of a PPA pixel is different from that of the optic disc. The optic disc pixel ratios are close to 1 while the ratios of the PPA pixels are greater than 1 . The final image contains the PPA pixels in white which can be eliminated from the optic disc.

\subsection{False-Region Elimination}

While approximating the optic disc area to an ellipse, it is possible that certain false region gets selected. In this case, a further threshold function needs to be used to eliminate the false regions and improve the accuracy of the algorithm

\section{CONCLUSION}

Peripapillary Atrophy detection and elimination is a significant factor for considering Glaucoma diagnosis. This paper describes a few existing methods for the same and proposes a new method for PPA detection. It segments a region of interest, approximates it to an ellipse using CEHT function and calculates the R/G ratio for each pixel within the ROI to detect the PPA pixels. The algorithm can be improved by considering another operation instead of subtraction for selecting the ROI. This can further help in eliminating the false regions and improve the accuracy.

\section{REFERENCES}

[1] H. A. Quigley, "Number of people with glaucoma worldwide," The British Journal of Ophthalmology, vol. 80, pp. 389-393, 1996.

[2] P. J. Evans, "The Underlying Cause of Glaucoma," The British Journal of Ophthalmology, pp. 745-783, 1939.

[3] P. Singh, "Tonometry: An Overview," IOSR Journal of Dental and Medical Sciences, vol. 13, Issue 1, Ver. 1, pp. 67-70, 2014.

[4] A. Colebrander, "Principles of ophthalmoscopy." In: Tasman W, Jaeger EA, eds. Duane's Ophthalmology.

[5] U. Schiefer, J. Pätzold, F. Dannheim, P. Artes, W. Hart, "Conventional Perimetry Part I: Introduction - Basic Terms," Der Opthalmologe, pp. 627-646, 2005.

[6] P. Singh, M. Tyagi, Y. Kumar, K. Kuldeep and P. Sharma, "Gonioscopy: A Review," Open Journal of Ophthalmology, Vol. 3 No. 4, 2013, pp. 118-121.
[7] C. Muramatsu, T. Nakagawa, A. Sawada, Y. Hatanaka, T. Hara, T. Yamamoto, and H. Fujita, "Determination of cup and disc ratio of optical nerve head for diagnosis of glaucoma on stereo retinal fundus image pairs," Proceedings SPIE Medical Imaging, vol. 7260, pp. 72603L-1-8, 2009.

[8] Y. Hatanaka, A. Noudo, C. Muramatsu, A. Sawada, T. Hara, T. Yamamoto, and H. Fujita, "Vertical cup-to-disc ratio measurement for diagnosis of glaucoma on fundus images," Proceedings SPIE Medical Imaging, vol. 7624, pp. 76243C-1-8, 2010 .

[9] C. Muramatsu, Y. Hatanaka, A. Sawada, T. Yamamoto, H. Fujita, "Computerized Detection of Peripapillary Chorioretinal Atrophy by Texture Analysis," 33rd Annual International Conference of the IEEE EMBS, August 30 - September 3, 2011.

[10] J. Cheng, J. Liu, D. W. K. Wong, F. Yin, C. Cheung, M. Baskaran, T. Aung, T. Y. Wong, "Automatic Optic Disc Segmentation with Peripapillary Atrophy Elimination," 33rd Annual International Conference of the IEEE EMBS, August 30 - September 3, 2011.

[11] J. Cheng, J. Liu, D.W.K. Wong, N. Tan, C. Cheung, M. Baskaran, T. Y. Wong, Seang Mei Saw, "Peripapillary atrophy detection by biologically inspired feature," Pattern Recognition (ICPR), $2012 \quad 21 \mathrm{st}$ International Conference on, vol., no., pp.53,56, 11-15 Nov. 2012.

[12] J. Cheng, D. Tao, J. Liu, D. W. K. Wong, N. Tan, T. Y. Wong, S. M. Saw, "Peripapillary Atrophy Detection by Sparse Biologically Inspired Feature Manifold," Medical Imaging, IEEE Transactions on, vol.31, no.12, pp.2355,2365, Dec. 2012.

[13] J. B. Jonas, M. C. Fernández, "Shape of the neuroretinal rim and position of the central retinal vessels in glaucoma," The British Journal of Ophthalmology, 1994;78(2):99-102.

[14] S.D. Ţălu, Ophtalmologie - Cours, Medical Publishing House Iuliu Haţieganu, Cluj-Napoca, Romania, 2005.

[15] S. Talu, M. Talu, Z. Fazekas, S. Giovanzana, "Multifractal analysis of human peripapillary atrophy," Image and Signal Processing and Analysis (ISPA), 2013, 8th International Symposium on, vol., no., $\begin{array}{llll}\text { pp.705,710, } & \text { 4-6 } & \text { Sept. }\end{array}$ 